ENZYMES

\section{Proproteuse Prospects}

from our Molecular Biology Correspondent

Ix seems to be a general rule of nature that animal proteases are manufactured in a harmless packaged form, as zymogens, and are activated, generally by the intervention of some other protease, when the organism has need of them. This process may have evolved in part as a device to prevent the onset of a lethal proteolytic activity on completion of biosynthesis, and, more interestingly, as a refined control mechanism, as reflected, for example, in the role of the enzyme cocoonase. This is a serine protease, produced by the silk moth for use as a chemical crowbar to break open its cocoon. It is synthesized as a zymogen, prococoonase, in cells at the mouth of the insect, is deposited on the surface, where it forms a solid crystalline crust, is activated, and then dissolved in a specially secreted potassium bicarbonate solution, with a $\mathrm{pH}$ corresponding to the activity optimum. The solution seeps into the cocoon, and dissolves out the sericin, a proteinaceous "silk glue", and leaves the moth free to pass into the outside world.

The latest in a series of articles from the two laboratories which have uncovered this scheme (Berger, Kafatos, Felsted and Law, J. Biol. Chem., 246, 4131; 1971) now describes the nature of the zymogen, and the manner of its activation. An extract of the secretory parts of the insect, at a point in its development some days before emergence, contains no cocoonase activity. The attempts first made at activation of the prococoonase in this solution with active cocoonase resulted in rapid loss of the activity of the latter. This, it transpired, was a consequence of attack by a tyrosinase present in the blood. When this enzyme was inhibited the cocoonase was seen to bring about gradual activation. Trypsin or subtilisin, on the other hand (but not chymotrypsin), caused very rapid liberation of the full cocoonase activity, and it was therefore surmised that free cocoonase can play only a secondary role in activation. One does not, however, have to search far for a source of activating protease activity: at an early stage of adult development the region between the adult and the pupal cuticle is full of a liquid, the moulting fluid, rich in proteases, which serves to digest the pupal cuticle. When the fluid has done its work it is reabsorbed, leaving apparently a residuum of proteases to look after the prococoonase.

Estimates of molecular weights by a number of methods showed that the molecular weight of the zymogen was about 32,000 , some $8-10,000$ more than free cocoonase. Thus by contrast with trypsin, which it otherwise closely resembles, a large part-about one quarter- of the chain is lost on activation. Benger et al. point out that because the activation fragments of zymogens are in effect nothing more than covalently bound inhibitors, the structural requirements may be supposed to be much less stringent than for the active enzyme, and that large differences introduced in the course of evolution in these parts of the chain are not perhaps so very surprising.

Another new zymogen has been discovered by Harper, Bloch and Gross (Biochemistry, 10, 3035; 1971). This is the precursor of the collagenase which the tail of the tadpole carries, as the means for its own annihilation when the moment of metamorphosis is at hand. Collagenases have been widely identified in animal tissue, and especially in diseased conditions, in which the elimination of collagen masses is desirable. The proenzyme was discovered through the observation that anti-collagenase antibodies were absorbed by collagenase-free extracts of tadpole tail-fin tissue. The chromatographic fraction of the extract believed to contain the zymogen failed to generate collagenase activity on treatment with trypsin or chymotrypsin. When, however, the fraction was mixed with a medium in which living tail-fin tissue had been maintained for some days, and incubated at $37^{\circ} \mathrm{C}$, activation ensued. The collagenase could be detected by its effect on the viscosity of the substrate, and by the appearance of tropocollagen fibrils with characteristic periodicities in the electron microscope. The collagenase produced under these controlled conditions was identified with the usual preparations by its inhibition by anti-collagenase antibodies. The collagenase has apparently the remarkably high molecular weight (assuming the reliability of the detergent-gel electrophoresis method) of 110,000 , or so, and the zymogen seems to be slightly larger. The activating factor is easily inactivated by heat, and is evidently a specific protease, that appears only at the right moment.

\title{
Precursors of DNA Replication
}

THE biochemistry of DNA replication as opposed to DNA repair is still a great enigma and, according to Werner, so it will remain if those intent on elucidating it persist in assuming that DNA replicase uses the deoxynucleoside triphosphates as immediate precursors. For in next Wednesday's Nature New Biology, Werner describes experiments which lead him to the conclusion that the enzyme responsible for the semiconservative replication of DNA in Escherichia coli polymerizes some activated form of the deoxynucleoside monophosphates.

Earlier this year, Werner reported in Nature (230, 570; 1971) that short pulses of ${ }^{3} \mathrm{H}$-thymidine are incorporated preferentially in small DNA chains, Okazaki pieces, whereas short pulses of ${ }^{3} \mathrm{H}$-thymine are preferentially incorporated into larger DNA chains. From this he argued that $E$. coli can differentiate between thymidine and thymine using the former for repair DNA synthesis and the latter for replication. His latest experiments are an attempt to identify the pathway of incorporation of ${ }^{3} \mathrm{H}$-thymine into DNA. When there is a dynamic equilibrium between the intracellular nucleotide pools-a steady-state condition-the pool of thymidine monophosphate is maximally labelled about 1 min after the cells are exposed to ${ }^{3} \mathrm{H}$-thymine. Maximum incorporation of the thymine into DNA and maximal labelling of the pools of thymidine diphosphate and triphosphate are attained after about 10 min. TMP is therefore not itself the immediate precursor of DNA replication but either TDP or TTP might be.

When, however, the cells are fed ${ }^{3} \mathrm{H}$ thymine and simultaneously deoxyadenine or thymidine, which act as donors of deoxyribose, the pool of TMP is greatly increased and is maximally labelled within about $10 \mathrm{~s}$ and the maximal rate of incorporation of the thymine into DNA is reached within 20-30 s. The pools of TDP and TTP are, however, not maximally labelled until about $2.5 \mathrm{~min}$. From these observations and measurements of the specific activities of the pools of TMP, TDP and TTP Werner concludes that although TMP is not itself an immediate precursor of DNA replication it is on the direct pathway to replication while TDP and TTP are not, and has to be converted to an activated form of TMP before being incorporated into DNA.

Because the size of the pool of activated TMP precursor is much smaller than the pool of TTP, and because DNA is replicated so very rapidly Werner suggests that simple diffusion and collision would be too slow to support the selection and alignment of the activated monophosphate precursors by the DNA replicase. He speculates therefore that some protein like that described by Alberts and Frey (Nature, 227,$1313 ; 1970$ ) may unwind the DNA duplex ahead of the replicase. The exposed single strands can then act as templates for the preselection and alignment of the precursors and all the DNA replicase has to do is link them together by phosphodiester bonds. 